\title{
Temporal resampling of time-varying infrared images sequences
}

\author{
by R. Montanini*, T. Scimone*,S. De Caro* and A. Testa*
}

\author{
*Univ. Messina, C.da di Dio, I-98166Messina, Italy, \{rmontanini, tscimone, sdecaro, atesta\}@unime.it
}

\begin{abstract}
The paper presents an effective method for the measurement, using a standard infrared camera working at 25 $\mathrm{Hz}$ frame rate with $320 \times 256$ pixels full frame, of very fast temperature transients (i.e., having a bandwidth of $10 \mathrm{kHz}$ or more) which, in principle, would require a much faster acquisition rate. The proposed method is based on triggering multiple time-delayed acquisitions (MTDA) of the observed thermal phenomenon, which has to be reproducible, by means of a very precise and stable programmable digital micro-controller and by reconstructing the time domain IR sequence using the frames acquired at each trigger event. The method could find application in assessing the reliability of power electronic devices and, in particular, to measure dynamically temperature distribution over the source metal of Power MOSFETs or IGBTs, which feature very fast thermal transients, even in the hundreds of microsecond scale and might develop local hot spots as a consequence of aging or failures.
\end{abstract}

\section{Introduction}

The ability to track the thermal evolution of a semiconductor device as a response to an electrical excitation is of paramount importance for the characterization of power electronics equipment [1], since it provides the R\&D engineer with the necessary background information for the development stage, allowing key investigations, such as analysis of reliability [2-3], numerical modeling [4], technology enhancement and design optimization [5-6], to be carried out.

Often designers are more interested in achieving the complete $2 \mathrm{D}$ temperature distribution across a welldefined region of the device rather than inferring the average temperature of the device itself [7]. While in this last case electrical methods exploiting the temperature sensitivity of specific properties of semiconductor devices can be used (e.g., the forward voltage of a pn-junction at a constant current is known to vary with temperature in a predictable way), detection of temperature distribution either requires a single-point $x-y$ raster scanning system or the usage of an infrared camera. Moreover, whereas electrical methods make use of electrical connections which are already available for normal device operation and are the only type that can be made on fully packaged devices [7], measurement of the temperature distribution by means of contact or non-contact methods always requires an optical access to the device surface, which therefore must be preliminary opened.

The thermal excursions that power devices undergo in the transient regime can be very large and fast. In addition, significant thermal gradients can arise on the device surface because of non-homogeneous current density distribution, which in turn can cause thermal instabilities and device failure. Hence, in order to get the temperature distribution of the device surface, a measurement method with both broad bandwidth and proper spatial resolution is needed. The fulfillment of these requirements is not trivial. The problem has been faced by some research groups in the last few years and different interesting solutions have been proposed [1-2, 8-11], most of which are based on infrared sensing.

All the systems described so far rely on single-point measurements and hence require a proper scanning system in order to reconstruct the temperature map distribution over the region of interest. Modern infrared cameras with fast focal plane array (FPA) would in principle present definite advantages compared to single-point $x$ - $y$ raster scanning systems. Unfortunately, these devices have a common limitation regarding the maximum sampling rate at full frame, which is usually limited. Higher frame rates are sometimes possible at the expense of a reduced number of pixels. Recently, Riccio et al [12] used the equivalent time sampling (ETS) concept to measure transient temperatures with an equivalent bandwidth of $100 \mathrm{kHz}$ by means of a standard IR camera, thus allowing to overcome the limitations associated with single-point measurements as far as the observed phenomenon can be repeated periodically (as it is the case for power electronic devices heated by pulsed currents).

Here we exploit the same concept to propose an improved method that, in addition of allowing full frame measurements of replicable transient events with high bandwidth (limited only by the integration time of the IR camera) and good spatial resolution (depending on the optical lens used in the experimental set-up), also provides automatic compensation of the background temperature, thus eliminating systematic errors caused by ambient temperature shifts at each trigger event. The proposed method makes use of a precise and stable programmable digital micro-controller used to trigger multiple time-delayed acquisitions (MTDA), allowing the time domain infrared sequence to be reconstructed based on the time-delayed frames acquired.

To prove the effectiveness of the proposed MTDA method, experimental tests have been carried out on a planar resistor heated with repeated pulsed currents. A practical application dealing with a dynamical analysis of the temperature distribution over the source metal of an IGBT power device is finally presented. 


\section{The MTDA method}

A standard cooled IR camera (FLIR SC7000) equipped with a $320 \times 256$ pixels InSb FPA sensor was used for the experiments. The IR camera has a maximum frame rate at full resolution of about $100 \mathrm{~Hz}$, while the integration time can be set between $10 \mu \mathrm{s}$ and $20.000 \mu \mathrm{s}$, with steps of $1 \mu \mathrm{s}$. The integration time $(\tau)$ used for acquiring the infrared images actually determines the maximum equivalent sampling frequency $\left(f_{\mathrm{e}, \mathrm{max}}\right)$ that can be reached by the MTDA method, being:

$$
f_{e, \max }=\frac{1}{\tau}
$$

The measurement principle of MTDA is schematically illustrated in Fig.1. Signals used for IR image acquisition are generated by means of three digital ports of an ATmega328 programmable digital micro-controller. The trigger output port of the IR camera (trigger OUT) generates a time base constituted by a train of impulsive signals having a period equal to the sampling time of the IR camera. When a rising edge occurs in the trigger output, a signal is generated by the microcontroller and sent to the trigger input port (trigger IN) of the IR camera, starting the acquisition. A second signal is sent to the power device throughout a gate driver to start the heating event. These two signals are synchronized using the time base of the IR camera, thus providing an effective matching between the images acquisition and the transient event.

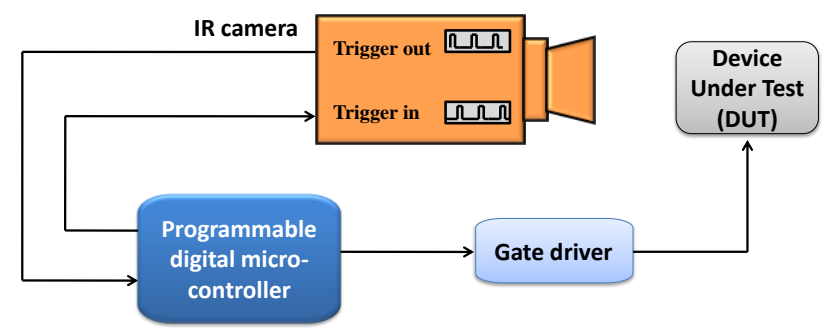

Fig.1.Schematic illustration of the MTDA method

To account for possible variation of the ambient temperature during the test (actually the number of cycles required for the reconstruction of the thermal transient can be quite long, depending on the dynamics of the event), a further single frame acquisition is triggered at the end of each cooling period and used for ambient temperature compensation. Hence, temperature increments over the initial temperature (delta_T) before the start of each heating cycle were considered. All the frames acquired at each trigger event are used at the end of the measurement procedure to reconstruct the time and space behavior of the temperature over the device.

If FR is the frame rate of the camera, at each iteration $N$ infrared images will be acquired (note that, due to the time delay, sometimes one image lies outside the duration of the event and can't be utilized for the reconstruction):

$$
N=D \times F R
$$

where $D$ represents the time duration of the transient event. The number of iterations depends on the time delay, and hence on the required equivalent sampling frequency. If $f_{\mathrm{e}}$ is the equivalent sampling frequency and $d t$ the time delay (i.e., the equivalent sampling time), then the number of iterations will be:

$$
M=\frac{f_{e}}{F R}
$$

Each iteration is shifted of $d t$ allowing the complete reconstruction of the transient event that must be replicated $M$ times. This holds as long as $D$ is greater than the frame rate (i.e., for slow transient events).

The timing sequence is schematically illustrated in Fig. 2 , in which a) represents the switching time of the device under test, b) the timing of images acquisition and c) the trigger signals used for synchronization. 


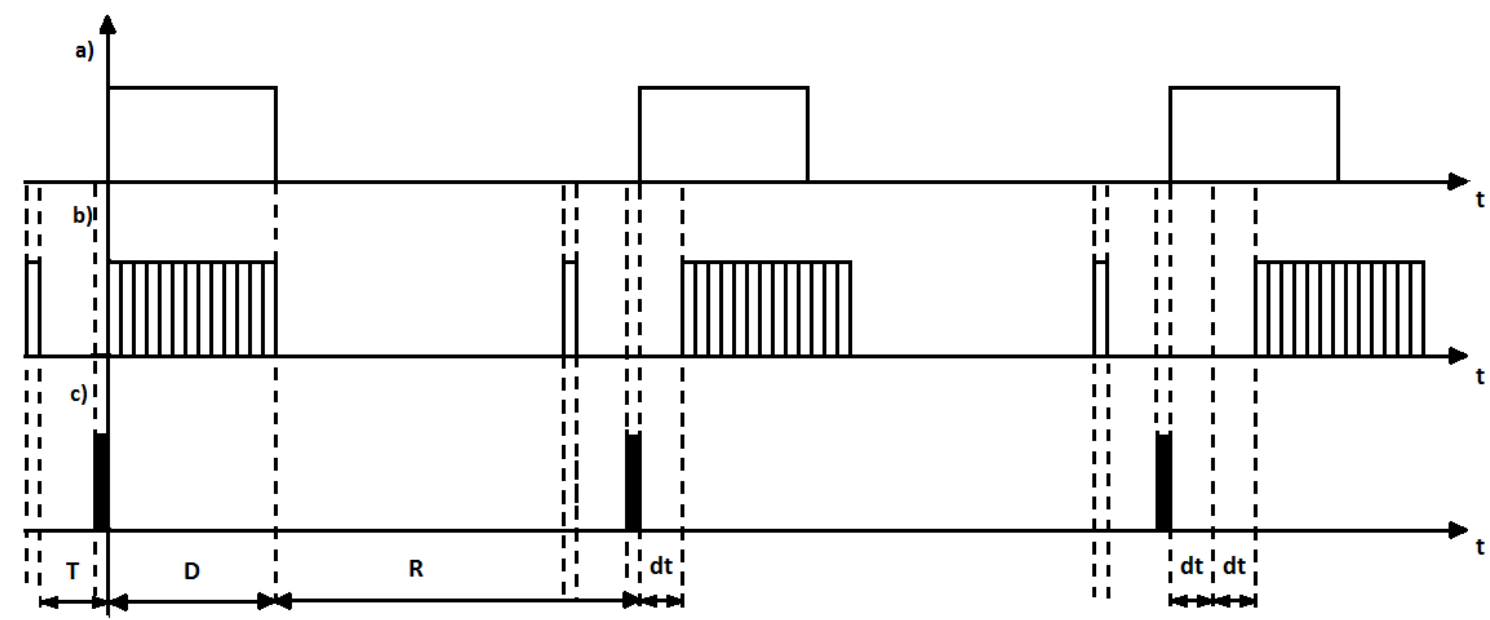

Fig. 2. Schematic illustration (not in scale) of the working principle of MTDA for slow transient events: $D=$ heating time, $R$ = repetition rate (cooling time), $d t=$ time delay, $T=$ pre-trigger used for ambient temperature compensation.

For fast transient event (i.e., when $D$ is smaller than the actual frame rate of the IR camera), the MTDA method can be still used by acquiring, at each iteration, only a single image instead of $N$ images (Fig. 3). In this figure the shaded rectangle actually represents the IR camera integration time, which, according to (1), must be smaller than the time duration of the event. Of course, in this case the duration of the reconstruction process will be much longer.

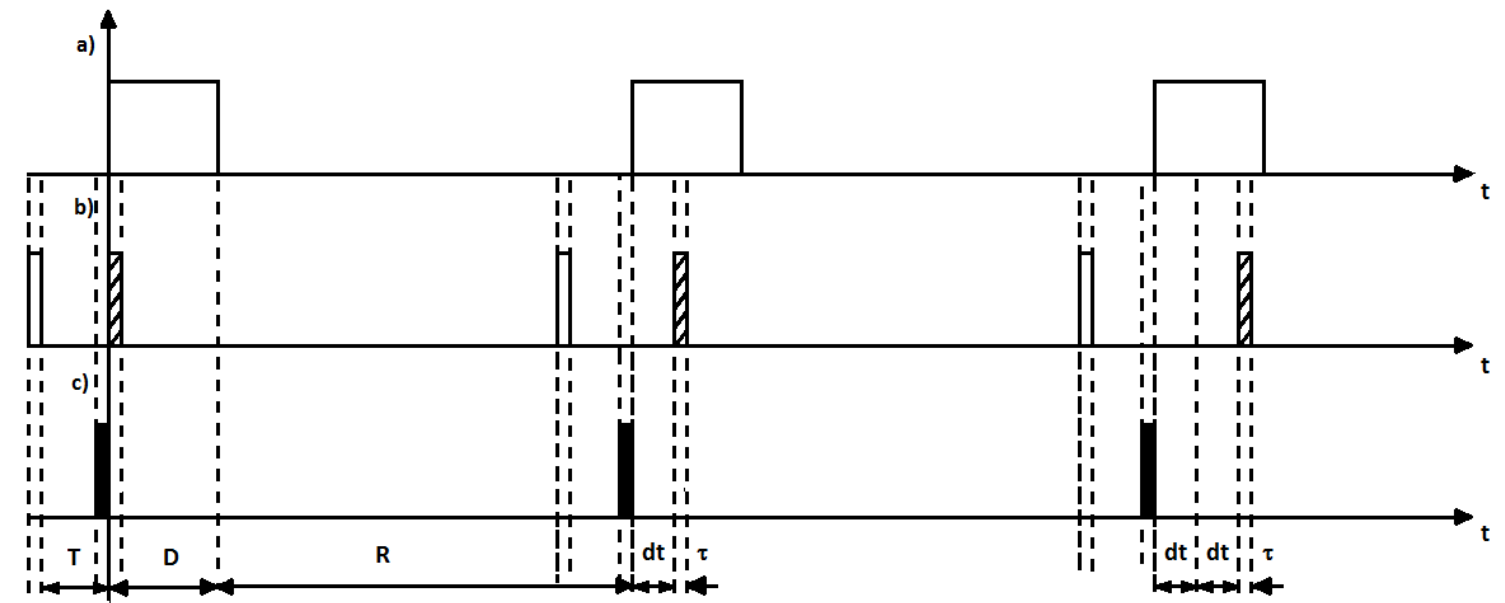

Fig. 3. Schematic illustration (not in scale) of the working principle of MTDA for fast transient events: $D=$ heating time, $R$ = repetition rate (cooling time), $d t=$ time delay, $T=$ pre-trigger used for ambient temperature compensation, $\tau=$ integration time.

\section{Measurements on a calibrated planar resistor}

As target, a laser-cut planar resistor with $1 \mathrm{~A}$ nominal current was used. The resistor was heated cyclically from ambient temperature up to about $37^{\circ} \mathrm{C}$ by means of pulsed currents of $D=1600 \mathrm{~ms}$ duration. The test circuit is reported in Fig. 4. No paint has been applied onto the resistor to uniform the target area emissivity, which was estimated to be about 0.95 by separate tests. The repetition rate of the trigger pulse (R) has been chosen to be 1 min in order to allow the device to dissipate the excess temperature increase before a new trigger pulse is applied. A further single frame acquisition is triggered at the end of each cooling period and used for ambient temperature compensation, as already explained in the previous section. All the frames acquired at each trigger event are used at the end of the measurement procedure to reconstruct the time and space behavior of the temperature over the device. All computations were carried out using Matlab ${ }^{\mathrm{TM}}$.

Fig. 5 shows infrared images taken at $25 \mathrm{~Hz}$ frame rate during one heating cycle (the complete sequence includes 40 images). These images were taken without introducing any time delay (i.e., $d t=0 \mathrm{~ms}$ ) and served as reference for verifying the effectiveness of the proposed method and assessing the measurement error of the reconstruction process. As expected, it can be observed that heat is generated suddenly as the current flows into the resistor, and eventually spread across the whole area of highly conductive material, with the hottest elliptically-shaped region located in the inner part of the resistive element. 


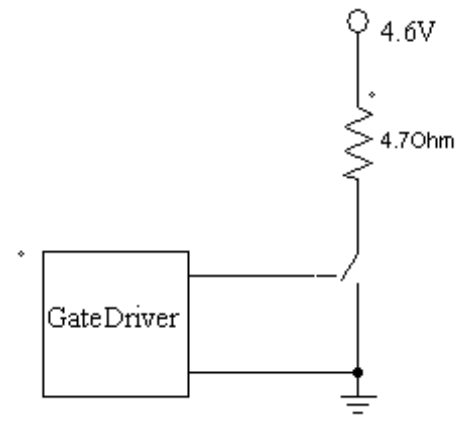

Fig. 4. Test circuit with resistor (slow transient events).

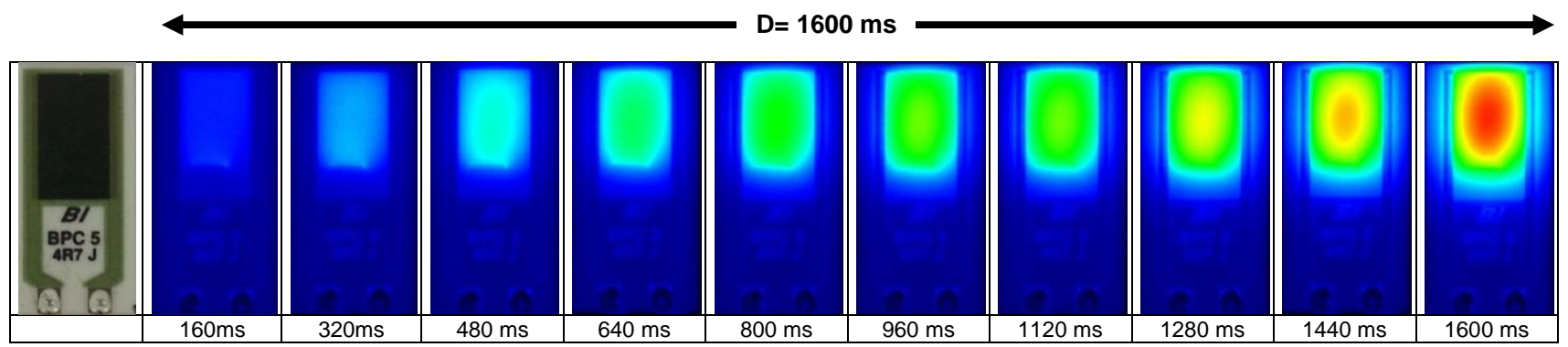

Fig. 5. Current-driven heating of the planar resistor (one cycle).

The next figure (Fig. 6) reports the temperature profile (delta_ $T$ increments) of the hottest point of the resistor measured throughout five cycles, with no time delay. From this plot it can be realized that the repeatability of the observed event is quite good, being the average standard deviation (21 mK) comparable to the NETD value of the used IR camera.

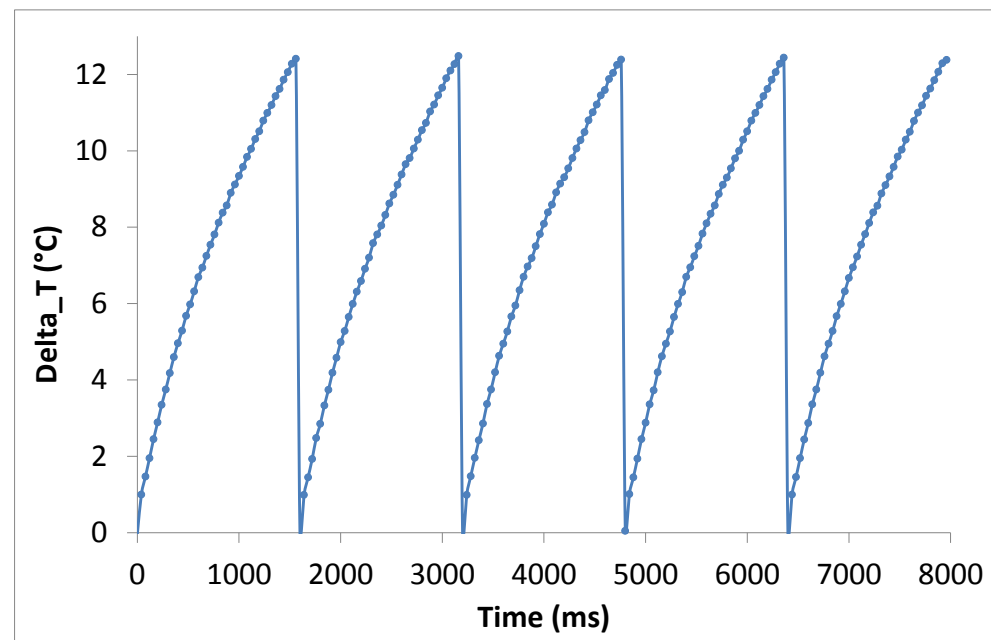

Fig. 6. Temperature profile of the hottest point of the planar resistor (five cycles, $d t=0 \mathrm{~ms}$ ).

The next experimental tests were carried out by using the MTDA method to capture the current-driven heating of the resistor with an equivalent sampling rate of $200 \mathrm{~Hz}(\mathrm{dt}=5 \mathrm{~ms})$ and $1000 \mathrm{~Hz}(\mathrm{dt}=1 \mathrm{~ms})$, respectively. In the first case, the complete sequence includes 320 infrared images, while in the second one the number of infrared images captured is 1600 . Each test was repeated five times and the average heating profiles of selected points of the target area were computed. Fig. 7 compares the reconstructed average heating profiles of the middle point (hot spot) of the target area with that obtained by using the infrared camera in normal operation mode (at $25 \mathrm{~Hz}$ frame rate). It can be observed a very good agreement among the three profiles. 




Fig. 7. Reconstructed heating profiles of the middle point of the target area, resampled at $200 \mathrm{~Hz}(\mathrm{dt}=5 \mathrm{~ms}$ ) and $1000 \mathrm{~Hz}(\mathrm{dt}=1 \mathrm{~ms})$. Values were measured by means of a cooled infrared camera operating at $25 \mathrm{~Hz}$ frame rate.

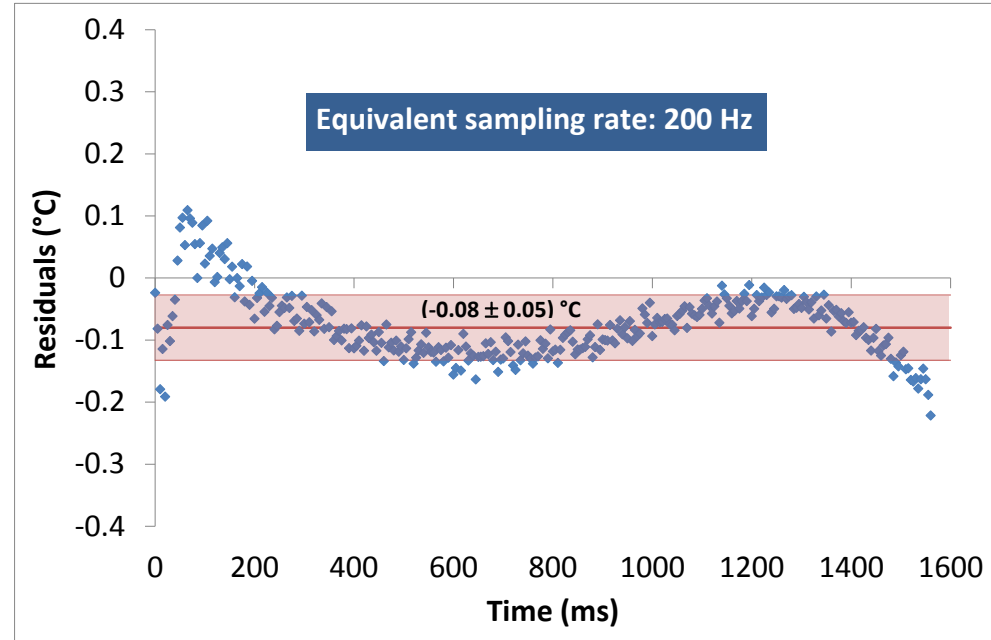

Fig. 8. Statistical deviation between average values of the heating profile of the middle point of the target area sampled at $200 \mathrm{~Hz}$ and third order polynomial fitting of the heating profile measured in normal operation mode.

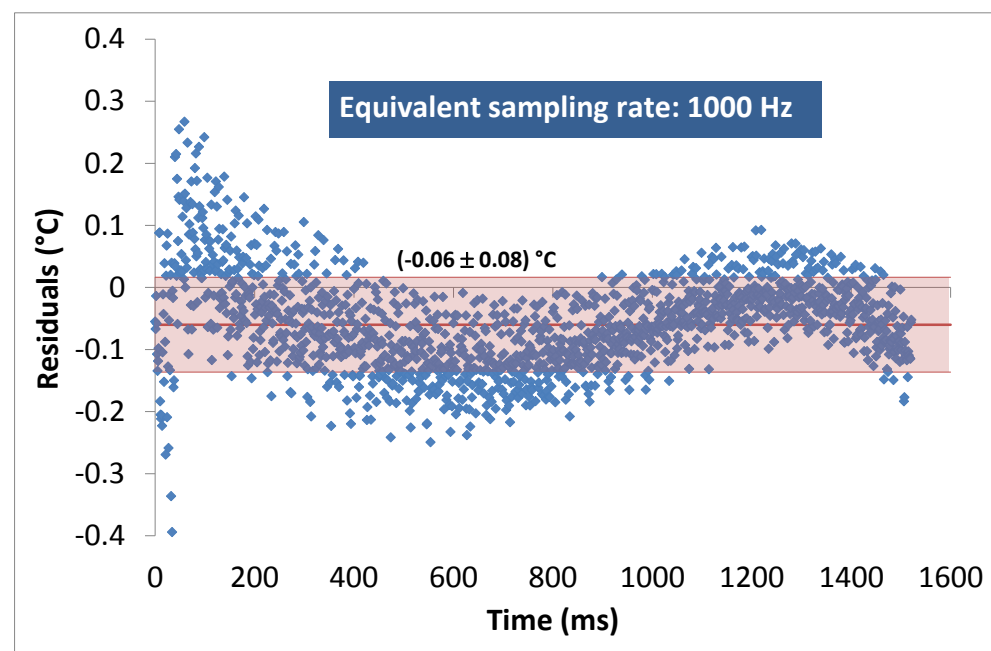

Fig. 9.Statistical deviation between average values of the heating profile of the middle point of the target area sampled at $1 \mathrm{kHz}$ and third order polynomial fitting of the heating profile measured in normal operation mode. 
In order to assess the measurement error of the MTDA method, the statistical residuals between the average values of the reconstructed heating profiles and the third order polynomial fitting of the heating profile measured in normal operation mode were evaluated. Results are shown for an equivalent sampling rate of $200 \mathrm{~Hz}$ and $1000 \mathrm{~Hz}$ in Figs. 8 and 9 , respectively. An average error of $(-0.08 \pm 0.05){ }^{\circ} \mathrm{C}$ and $(-0.06 \pm 0.08){ }^{\circ} \mathrm{C}$ was obtained, with no evidence of systematic effects related to the increasing of the equivalent sampling rate.

\section{Application to reliability assessment of an IGBT power device}

The on-state voltage of power electronic devices tasked to perform several billions of repetitive switching operations during their life is subject to modifications caused by the progressive ageing of semiconductor and metal layers. Endurance tests are the traditional way to monitor these changes in order to assess device reliability. However, they are very time expensive, requiring even months of uninterrupted laboratory tests. An interesting alternative is to assess the reliability through a suitable mathematical model. Specifically, coupling the results of a very fast thermodynamic analysis with a reliability model based on the Coffin-Manson law, device degradation over time can be estimated and the level of reliability evaluated [10]. As an example of application, the proposed MTDA method was applied to investigate the temperature distribution over the source metal surface of an IGBT power device during a switching cycle. According to the test circuit of Fig. 10, in each switching cycle the device is turned on for 6 ms to generate a fast heating, and then turned off for $60 \mathrm{~s}$, a time sufficient to allow the device to reach again the initial temperature. A $1.6 \mathrm{mH}$ inductor is exploited to limit the device current during the switching cycle. The device under test was previously subjected to a suitable accelerated ageing procedure, then, the package molding was chemically removed to expose the source metal surface, as shown in Fig. 11a.

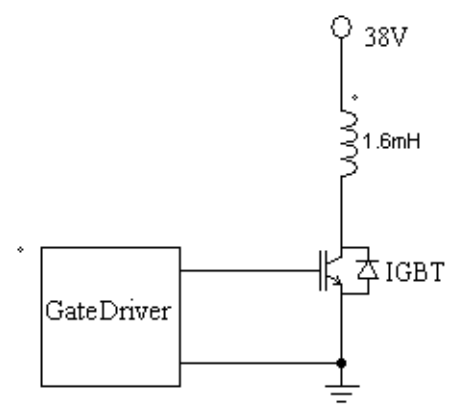

Fig. 10. Test circuit for IGBT fast dynamics analysis.

Since the exposed surface encompasses regions made of different materials (aluminum, oxides, passivation etc.), a suitable emissivity map has been carried out to obtain the true temperature mapping. This was done by passively heating the device by means of a heater. Moreover, in order to focus the investigation exclusively on the region of interest, captured images were post-processed by applying a mask exploiting the different emissivity of the target area with respect to the outer part of the device, which is made of silicon oxide. The time delay was set to $100 \mu$ s, therefore it was necessary to carry out 60 iterations to completely reconstruct the heating event of the device. Before further computation, each infrared image was masked and corrected for the actual emissivity. No microscope lens was used for this test, hence the spatial resolution is poor because of the small dimension of the device.

Fig. $11 \mathrm{~b}$ shows the reconstructed temperature distribution over the DUT surface when the maximum heating of the device occurs. It can be observed that some hot spots are present (as already explained, a device previously subjected to accelerated ageing was intentionally chosen for these tests), while gradients up to $10^{\circ} \mathrm{C}$ are clearly visible.
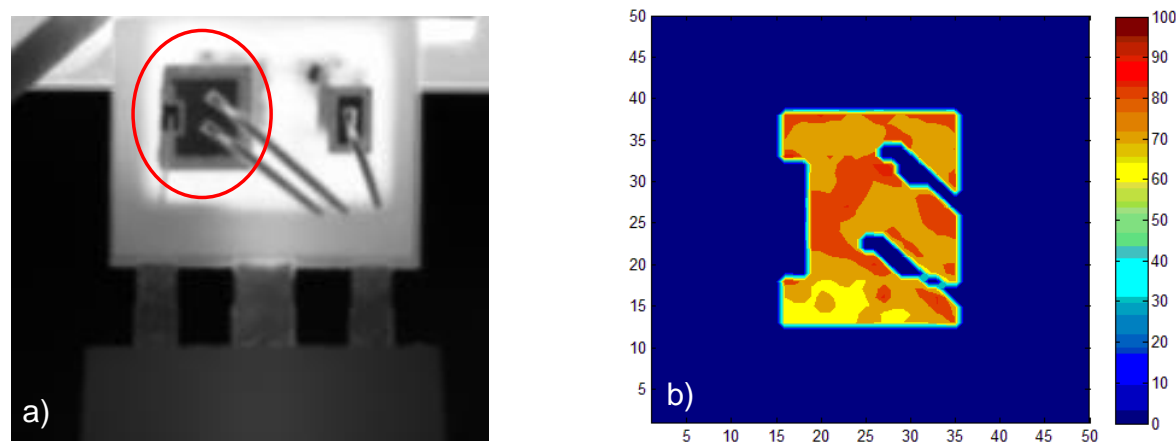

Fig. 11. a) Live image of DUT and b) reconstructed temperature distribution map sampled at $10 \mathrm{kHz}$ equivalent frame rate $(d t=100 \mu \mathrm{s})$. 


\section{Conclusions}

A novel method able to extend the area of application of standard infrared systems to the investigation of very fast heating/cooling transient events has been presented. The method basically relies on triggering multiple time-delayed acquisitions (MTDA) of the observed thermal phenomenon, by means of a very precise and stable programmable digital micro-controller and by reconstructing the time domain IR sequence using the frames acquired at each trigger event. As a result, the time resolution of full frame IR sequences is increased by two order of magnitude or more with respect to that of a standard infrared camera. Preliminary results obtained on a power electronics device with the molding package removed are very promising. The presented method has been originally developed to assess the reliability of power devices, but it could find application in other areas as well.

\section{REFERENCES}

[1] Castellazzi A., Honsberg-Riedl M. and Wachutka G., "Thermal characterization of power devices during transient operation", Microelectronics Journal, vol. 37, pp. 145-151, 2006.

[2] Irace A., Breglio G., Spirito P., R. Letor, S. Russo, "Reliability enhancement with the aid of transient infrared thermal analysis of smart Power MOSFETs during short circuit operation", Microelectronics Reliability, vol. 45, pp. 1706-1710, 2005.

[3] Yin C.Y., Lu H., Musallam M., Bailey C. and Johnson C.M., "In-service reliability assessment of solder interconnect in power electronics modules", Proceedings of Prognostics \& Systems Health Management Conference, paper MU3095, Macau, 5 pp., 2010.

[4] Breglio G., Rinaldi N., Spirito P., "Thermal mapping and 3D numerical simulation of new cellular power MOS affected by electro-thermal instability”, Microelectronics Journal, vol. 31, pp. 741-746, 2000.

[5] Breitenstein O., Langenkamp M., "Lock-in contact thermography investigation of lateral electronic inhomogeneities in semiconductor devices", Sensors and Actuators A, vol. 71, pp. 46-50, 1998.

[6] Riccio M., Rossi L., Irace A., Napoli E., Breglio G., Spirito P., Tagami R., Mizuno Y., "Analysis of large area Trench-IGBT current distribution under UIS test with the aid of lock-in thermography", Microelectronics Reliability, vol. 50, pp. 1725-1730, 2010.

[7] Blackburn D.L., "Temperature measurements of semiconductor devices - A review", in Proceedings of the 20th Annual IEEE Symposium on Semiconductor Thermal Measurement and Management, San Jose, California, pp. 70-80,2004.

[8] Rossi L., Breglio G., Irace A., Spirito P., "Thermal transient mapping systems for integrated semiconductor devices and circuits", in Proceedings of 8th Quantitative InfraRed Thermography conference, paper QIRT2006-022 Padua(Italy), 2006.

[9] Breglio G., Spirito P., "Experimental detection of time dependent temperature maps in power bipolar transistors", Microelectronics Journal, vol. 31, pp. 735-739, 2000.

[10] Testa A., De Caro S., Russo S., "A Reliability Model for Power MOSFETs Working in Avalanche Mode Based on an Experimental Temperature Distribution Analysis". IEEE Transaction on Power Electronics, vol. 27, pp. $3093-3100,2012$.

[11] Testa A., De Caro S., Panarello S.,Patanè S., Russo S., Patti D., Poma S, Letor R., " Reliability of planar, Super-Junction and trench low voltage power MOSFETs”. Microelectronics Reliability, vol. 50, pp. 1789 1795, 2010

[12] Riccio M., Breglio G., Irace A. and Spirito P., "An equivalent time temperature mapping system with a fullframe sampling rate", Rev. Sci. Instrum., vol 78, n.10, pp. 106106, 2007. 